\title{
Una aproximación al senado colombiano desde la biopolítica
}

\author{
An Approach to the Colombian Senate from Biopolitics \\ Uma abordagem ao senado colombiano a partir da biopolítica
}

DOI: https://doi.org/10.21803/penamer.13.26.421

Daniela Fernanda García Gamboa

https://orcid.org/0000-0002-2363-014X

Luisa Fernanda Patiño Marin

https://orcid.org/0000-0001-8390-8767

\section{¿Cómo citar este artículo?}

García, D. \& Patiño, L. (2020). Una aproximación al senado colombiano desde la biopolítica. Pensamiento Americano, 13(26), 63-75

DOI: https://doi.org/10.21803/penamer.13.26.421

\section{Resumen}

A través de los años el término biopolítica ha adoptado dos significados contrapuestos, uno basado en el filósofo Michael Foucault, el cual asocia este concepto con el control de la sociedad, es decir, cómo desde el poder político se puede estructurar y controlar la vida humana. El otro relacionado con una subdisciplina que tiene como base incorporar las ciencias de la vida con las ciencias políticas; comparando los diferentes fenómenos y comportamientos políticos apoyándose en la biología, la genética, teorías evolucionistas, neurociencias, entre otras, con el propósito de comprender mejor los diversos fenómenos políticos. A partir de la anterior aclaración el siguiente artículo pretende analizar el Senado colombiano (20142018) desde el enfoque biopolítico, examinando el comportamiento de los partidos políticos que lo conforman, comparándolo con diferentes conductas animales, con el objetivo de utilizar una perspectiva novedosa y poco implementada en el campo de la Ciencia Política.

Palabras clave: Biopolítica, Comportamiento animal, Partido político, Senado.

\begin{abstract}
Regarding biopolitics, it has adopted two contrasting meanings, one based on the philosopher Michael Foucault, which associated this concept with control, that is, how from political power human life can be structured and controlled. On the other hand, biopolitics have also been defined as a subdiscipline that has the basis of incorporating life sciences with political science, comparing the different phenomena and political behaviors based on biology, genetics, evolutionary theories, neurosciences, among others, aiming at a better understanding OF the various political phenomena. From the previous clarification the following article points out analyzing the Colombian Senate (2014-2018) taking into account the biopolitical approach, examining the behavior of the political parties that have been taken part in, comparing it with different animal behaviors, with the aim of using a novel and poorly implemented perspective in the field of Political Science.
\end{abstract}




\section{Resumo}

Ao longo dos anos, o termo biopolítica tem adotado dois sentidos opostos, um deles baseado no filósofo Michael Foucault, que associa esse conceito ao controle da sociedade, ou seja, como a vida humana pode ser estruturada e controlada a partir do poder político. A outra relacionada a uma subdisciplina baseada na incorporação das ciências da vida às ciências políticas; comparar os diferentes fenômenos e comportamentos políticos com base na biologia, genética, teorias evolutivas, neurociências, entre outros, a fim de melhor compreender os vários fenômenos políticos. Com base no esclarecimento anterior, o seguinte artigo procura analisar o Senado colombiano (2014-2018) a partir do foco biopolítico, examinando - comportamento dos partidos políticos que o compõem, comparando-o com diferentes comportamentos animais, com o objetivo de utilizar um romance. perspectiva e pouco implementada no campo da Ciência Política.

Palavras-chave: Biopolítica, Comportamento animal, Partido político, Senado.

\section{Perfil}

Profesional en Relaciones Internacionales y Estudios Políticos, Universidad Militar Nueva Granda. Email: danielagarciagamboa98@gmail.com

Profesional en Relaciones Internacionales y Estudios Políticos, Universidad Militar Nueva Granda. Email: Iusiafernandap@gmail.com
Daniela Fernanda García Gamboa

Luisa Fernanda Patiño Marin 


\section{Introducción}

La biopolítica se ha convertido con el pasar de los años en una herramienta que ha permitido interpretar la política desde una perspectiva diferente, pero con el tiempo se ocasionó una gran confusión con respecto a ¿Qué es la biopolítica?, dado que se han generado definiciones contrapuestas, como resultado este término se ha asociado con dos perspectivas diferentes: primero, con el pensamiento de Foucault enfocado en el control de la sociedad y segundo, con la política científica la cual explica el comportamiento político por medio de la biología.

A partir del enfoque Biopolítico se busca realizar una comparación entre el comportamiento de los animales, el cual se basa en los estímulos recibidos en el medio ambiente en el que habitan, y con la conducta presentada en el Senado colombiano. El objetivo de esta investigación es identificar el comportamiento de los partidos políticos durante el periodo 2014-2018 teniendo en cuenta el ambiente político, el clientelismo y el jefe de estado del momento, con el fin de dar respuesta al ¿́por qué? de las acciones tomadas por los partidos políticos y sus motivaciones.

Cabe resaltar que el estudio de la política colombiana específicamente de los órganos estatales es un tema escasamente analizado, como resultado se ha dado la poca generación de nuevo conocimiento acerca de cómo funcionan, para qué sirve y cómo están estructurados los diferentes órganos presentes en el Estado Colombiano.

Por otra parte, es importante la implementación de metodologías poco convencionales o utilizadas en la ciencia política, debido a que esto permitiría tener nuevas interpretaciones de los fenómenos políticos, de las instituciones, de los actores y del ambiente político; por ende, se da la creación de nuevo conocimiento, el cual es la herramienta para poder tener mejor entendimiento de la política desde diferentes perspectivas.

En concordancia con lo anteriormente expues- to el artículo se estructura de la siguiente forma: En primera instancia se hace una breve explicación al interrogante de ¿qué es la biopolítica? asociada con el pensamiento de Foucault y la biopolítica científica. Luego se analizará la figura del Senado dentro del estado colombiano. Posteriormente se realizará una breve explicación del Senado colombiano durante el periodo 2014-2018. Finalmente se presentarán los resultados producto de la comparación entre los comportamientos de los distintos partidos políticos que conforman el Senado colombiano con los comportamientos de los animales.

\section{Metodología}

El siguiente artículo es una investigación cualitativa de tipo exploratoria, que desde la disciplina de la biopolítica pretende interpretar el comportamiento de los partidos políticos colombianos que pertenecieron al Senado en el periodo 2014-2018. Este enfoque se basa en cómo existe una relación entre la conducta de los seres humanos tanto individual como en sociedad con los comportamientos de los animales; el fin de esta comparación es poder comprender la estrecha relación entre el comportamiento animal y el comportamiento político.

\section{¿Qué es la biopolítica?}

Cuando se habla de biopolítica se tiende a asociar con el pensamiento de Michael Foucault, el cual establece que esta disciplina se encarga de analizar cómo el poder social y político se utiliza para controlar la vida humana; para Foucault la historia no es una expresión continua de dominadores y dominados, sino que la historia se ve reflejada a través de la sociedad, el lenguaje, las normas, las instituciones,entre otras; el fin de esta perspectiva de biopolítica es la regulación de la población en todos sus ámbitos. Además, habla sobre el concepto de biopoder que es la manera de ejercer fuerza sobre la población buscando maximizar sus fuerzas e integrarlo en sistemas eficientes.

Según Paul Rabinow y Nikolas Rose (2006), aclaran cuál es la relación entre biopoder y biopolítica en 
el mundo contemporáneo. En primer lugar, se refiere a biopoder a algo que se puede atraer por medios más o menos racionalizados de poder intervenir sobre las características "vitales de la existencia humana" (Rabinow \& Rose; 2006, p.197). Además, Giorgio Agamben \& Antonio Negri afirman que el biopoder toma una forma en donde la política depende de la dominación y/o eliminación de la existencia vital de algunos sujetos que la ejercen.

Por último, Paul Rabinow \& Nikolas Rose hablan sobre la biopolítica, como un compendio de estrategias específicas sobre las problemáticas de la vitalidad humana, la morbilidad y la mortalidad; "sobre las formas de conocimiento, los regímenes de autoridad y las prácticas de intervención que son deseables, legítimas y eficaces." (Rabinow \& Rose; 2006, p.197) Para Hardt \& Negri argumentan que la biopolítica se puede ver como un ejercicio de poder que regula la vida social desde su interior, controlando las conciencias y cuerpos de la población (Herrera Burton, 2018).

Antes de que Michael Foucault acuñara el término de biopolítica, los politólogos como Rudolf Kjellén, aplicaban esta disciplina de manera distinta, no buscaban determinar cómo se podía generar un control sobre la sociedad, sino como existía una relación entre el comportamiento de los humanos de manera individual o en sociedad con los comportamientos animales (Gunneflo, 2015). Finalmente, el resultado que deseaban obtener era una mayor comprensión de la naturaleza humana y revitalizar el estudio del comportamiento político; así como buscar las contribuciones biológicas que le podía hacer a la disciplina.

Debido a esta diferencia de interpretación de la biopolítica, se generaron dos vertientes: la biopolítica foucaultiana y la biopolítica científica las cuales integran las ideas y los datos de las ciencias de la vida empírica y racionalista al estudio de la política, además, utilizan las teorías y los datos; la biopolítica científica se utiliza para realizar comparaciones y análisis del comportamiento de la sociedad.

Desde 1970 un grupo de politólogos adoptó for- malmente el término de biopolítica para definir su interés en la política y la biología. Entre estos politólogos que defendían la biopolítica científica se encontraba Somit \& Peterson, los cuales veían la biopolítica como el término que describe el enfoque interdisciplinario utilizado por los académicos el cual sostiene que los datos y teorías de la biología tienen una relación con el comportamiento político "la biopolítica es una orientación a la investigación política que reconoce a la persona como una criatura compleja, racional, emocional y biológica" (Somit \& Peterson 1998, p.562).

Estos politólogos examinaron la política como un fenómeno biológico, considerando variables biológicas y ambientales en su investigación, es decir, observando el comportamiento y las acciones repetitivas que se presentaban frente algún estímulo o cambio del ambiente. En este método se tiene en cuenta el hecho de que el ambiente y la cultura interactúan con el repertorio conductual del individuo, como explica Roger Masters:

El comportamiento humano es el producto de una integración, dentro del cerebro y el sistema nervioso de cada individuo, de información seleccionada filogenéticamente transmitida por los genes, información históricamente seleccionada transmitida por lenguaje, símbolos culturales y la información adquirida individualmente en el ciclo de vida. los individuos y la sociedad pueden evolucionar hacia nuevos patrones de comportamiento, del mismo modo que todas las especies incluida la nuestra. (Flohr,1990, p. 177)

\section{Senado de la República de Colombia}

Las ramas del poder público del Estado colombiano, según su constitución política (1991) son: legislativa, ejecutiva y judicial. La rama legislativa, es el Congreso de la República, el cual es bicameral, es decir, que está conformado por el Senado (cámara alta) y la Cámara de Representantes (cámara baja).

El Senado de la República de Colombia es un cuer-

Pensamiento Americano Vol. 13 - No. 26 - p.p. 63-75 • 2020 • Enero-Junio • Corporación Universitaria Americana • Barranquilla, Colombia • ISSN: 2027-2448 http://publicaciones.americana.edu.co/index.php/pensamientoamericano/index 
po colegiado de elección popular por circunscripción nacional elegido por periodos de cada cuatro años, en donde se cuenta el total de votos obtenidos por los candidatos en todo el país, en el que no importa su partido político, lo único que necesitan es superar el umbral electoral en las elecciones y mediante la cifra repartidora exceder un mínimo de votos que no deben ser inferior al $2 \%$ de los sufragados para el Senado, sin importar su orientación política y tampoco su relación frente al gobierno tanto de oposición o simpatía.

El senado está conformado por 102 personas que ocupan el puesto de senador, en el cual 100 senadores(as) son elegidos por voto popular en todo el territorio nacional y 2 senadores(as) son elegidos pertenecientes a la circunscripción especial indígena. (Senado de la República de Colombia, 2019) Pero a partir del Acto Legislativo 2 de 2015, el candidato que le siga en votos al presidente de la República ocupara un curul en el Senado y con la ley 1909 de 2018 se adiciona 5 senadores(as) que pertenezcan al partido Fuerza Alternativa Revolucionaria del Común.

De acuerdo con el Artículo 6 de la Ley 05 de 1992, decreta que el Congreso de la República de Colombia ejerce 8 funciones específicas las cuales son:

1. Función constituyente: Reformar la constitución por medio de actos legislativos 2. Función legislativa: Elaborar, interpretar, reformar y derogar las leyes y códigos en todos los ramos de la legislación. 3. Función de Control político: Solicitar y situar a los ministros del despacho y demás autoridades y conocer de las acusaciones que se formulen contra altos funcionarios del Estado. 4. Función judicial: Juzgar a los altos funcionarios del Estado por responsabilidad política. 5. Función electoral: Elegir Contralor General de la República, Procurador General de la Nación, Magistrados de la Corte Constitucional y de la Sala Jurisdiccional Disciplinaria del Consejo Superior de la Judicatura, Defensor del Pueblo y vicepresidente de la República, cuando hay falta absoluta. 6. Función administrativa: para establecer la organización y funcionamiento del Congreso Pleno, el Senado y la Cámara de Representantes. 7. Función de control público: Colocar a cualquier persona, natural o jurídica, a efecto de que rindan declaraciones, sobre hechos relacionados con las indagaciones que la Comisión adelante. 8. Función de protocolo: Recibir a Jefes de Estado o de Gobierno de otras naciones. (art 6)

De igual manera según los artículos 173 de la Constitución Política de Colombia (1991), son funciones del Senado admitir o no la renuncia bien sea del presidente y el vicepresidente de la República; aprobar o no los ascensos de oficiales de las Fuerzas Militares y pública; permitir el tránsito de tropas extranjeras por el territorio nacional; elegir al Procurador General de la Nación y los magistrados de la Corte Constitucional.

Por último, durante el periodo constitucional, en las dos cámaras funcionarán las Comisiones Permanentes, las Comisiones Legales, las Comisiones Especiales y las Comisiones Accidentales (Artículo 53 de la Ley 5 de 1992). Las Comisiones Permanentes trata temas específicos en el que se conforman grupos de congresistas con el fin de estudiar y votar en la diligencia de los proyectos de ley y actos legislativos, en este caso hay 7 Comisiones Constitucionales Permanentes que facilitan el trámite legislativo y de reforma constitucional. (Ley 3, 1992)

\section{El Senado de la República de Colombia 2014-2018}

El Senado de la República de Colombia, es elegido por votación popular por periodos de cada cuatro años; en el cual para el periodo 2014-2018 los resultados electorales del Senado según la MOE (2014) alcanzó un total de 14443055 de votos en el que fueron elegidos 102 senadores(as) de 9 distintos partidos políticos respectivamente. De acuerdo con la gráfica 1 hubo cuatro partidos políticos que abarcaron con la mayoría de los curules estos fueron: El Partido Social de Unidad Nacional (Partido de la U) (21), Centro Democrático (20), Partido Conservador Colombiano (18) y el Partido Liberal Colombiano (17). 


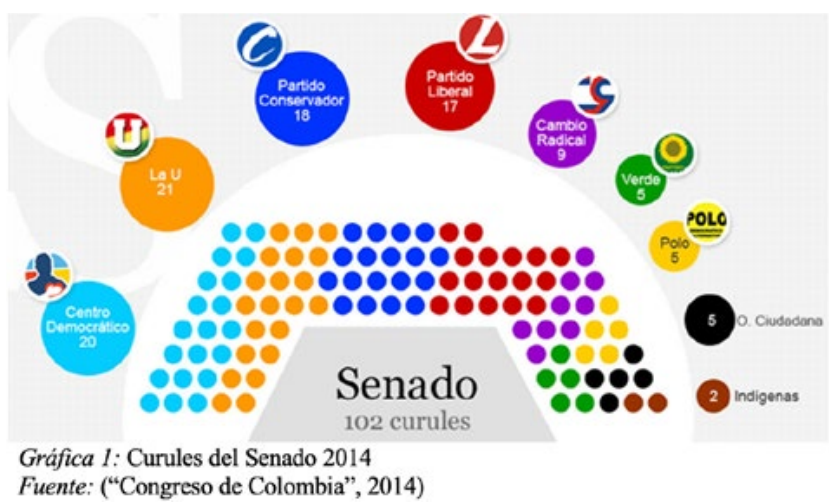

A partir de la anterior información se puede concluir que para el 2014 no se dio una mayoría consolidada, pues los votos se encontraron distribuidos entre los partidos como el de la Unidad Nacional (U), Centro Democrático, Liberal y Conservador. Este resultado representó una derrota parcial para el expresidente Juan Manuel Santos, porque a pesar del clientelismo que repartió en la Casa de Nariño y de contar con las ventajas del poder no logró consolidar una mayoría contundente del Partido Social de Unidad Nacional (U), la coalición que lo apoyó en los primeros 4 años de mandato.

Cabe anotar que según Rodríguez (2016), a lo largo de las administraciones del expresidente Santos se han visto diferentes comportamientos por parte de los partidos políticos. Por un lado están el partido Liberal, La U y Cambio Radical, los cuales han trabajado junto con el mandatario; por otro lado el Centro Democrático (derecha) y el Polo Democrático Alternativo (izquierda) cuyas posiciones han sido de oposición; además, el partido Conservador ha tenido acercamientos en algunas ocasiones mientras en otras no; por último, está el partido de la Alianza Verde que ha actuado de manera independiente, aunque de cierta manera este partido apoyó algunas ideas del gobierno del expresidente Santos pero aun así no dejaron de seguir sus ideales.

\section{Resultados}

\section{Conducta y ambiente}

Es poco conocido el inicio de la Biopolítica, por tal motivo se tiende a pensar que es una herramienta de análisis relativamente nueva, pero esto es un conocimiento erróneo dado que Aristóteles, utilizaba la comparación entre las acciones animales con el de las personas. Aristóteles sostiene que "los animales tienen voz, por medio de la cual manifiestan el dolor y el placer pero no logran significar lo perjudicial o lo conveniente, de forma que carecen de los conceptos del bien y del mal” (Neira, 2014, p. 262), lo cual señala que los animales tienen un entendimiento de su entorno y pueden manifestar inconformidades frente a este, a su vez también aplican una estructura de subordinación, donde el más fuerte debe garantizar la supervivencia del más débil, de forma semejante este tipo de organización se ve reflejada en el comportamiento de los humanos, el cual busca obtener el mayor beneficio por medio de la asociación al más poderoso.

El comportamiento de los animales puede tener fines alimentarios, migratorios, reproductivos, para marcar el territorio donde se desarrollan y también puede ser un comportamiento social (Marino, 1996). Además, es importante resaltar que el comportamiento de los seres vivos está determinado por los diferentes estímulos recibidos del medio ambiente en donde habitan. Por lo tanto, se considera que el ambiente es algo o alguien que rodea como pieza de su entorno (Real Academia Española, 2019), a raíz de esto la conducta de los animales está ligada al cambio de las condiciones tanto externas como internas, por ejemplo, cuando hay cambios en el clima que obligan a migrar a los animales o en el momento en que hay pérdida de alimento.

Teniendo en cuenta lo anterior y resaltando que existen diferentes conductas en el reino animal se realizará una comparación de las acciones de los partidos políticos que hicieron parte del Senado en el periodo 2014-2018 con los siguientes comportamientos animales:

1. Comportamiento del partido Social de la Unidad Nacional: Manada de elefantes

Los elefantes son animales dotados de inteligen- 
cia, que se articulan en una sociedad jerarquizada matriarcal; en donde la hembra dominante dirige el rumbo de la manada basando sus decisiones en la búsqueda de recursos hídricos, alimentarios y de seguridad (Candela, 2017). Por otra parte, estos mamíferos tienen un comportamiento nómada, pueden durar entre dos a tres semanas en el mismo lugar, esto depende de la abundancia o escasez de la comida (Borge, 2017). Una de las características más importantes de esta especie es su actitud despreocupada, siempre y cuando sus necesidades básicas estén cubiertas, de lo contrario; si su entorno cambia la manada pasa a tener una actitud protectora y seria con el fin de garantizar su supervivencia

Este tipo de comportamiento fue implementado por el partido de la U. El expresidente Juan Manuel Santos asumió un rol semejante al de la hembra alfa; evidenciando durante su mandato 2014-2018 un favoritismo hacia los integrantes de su partido; les brindó los mejores recursos políticos, se cita como ejemplo en el año 2014 el exmandatario defendió la llamada "mermelada" es decir el clientelismo que se estaba dando durante su mandato. Según el expresidente "La mermelada no es nada diferente a las inversiones que hace un gobierno con sus regiones" ("Santos salió en defensa", 2014), igualmente Santos aclaró que los senadores y congresistas tenían el derecho de sugerir inversiones e inversionistas en sus regiones.

Además, el exmandatario garantizó la supervivencia del partido, aumentando su influencia política principalmente en la opinión pública; esto se dio por medio del proceso de paz, debido a que el partido de la U fue uno de los líderes de esta propuesta y generando así que su impacto en la opinión pública a nivel nacional fuera cada vez mayor. Por otra parte, produjo que su influencia en los asuntos políticos fuera cada vez más grande. Por último, la actitud del partido durante este periodo fue despreocupada parecida a la de los elefantes, ya que tenían cubiertas la mayoría de sus necesidades políticas entre ellas: puestos estatales, contratos, influencia y poder político.

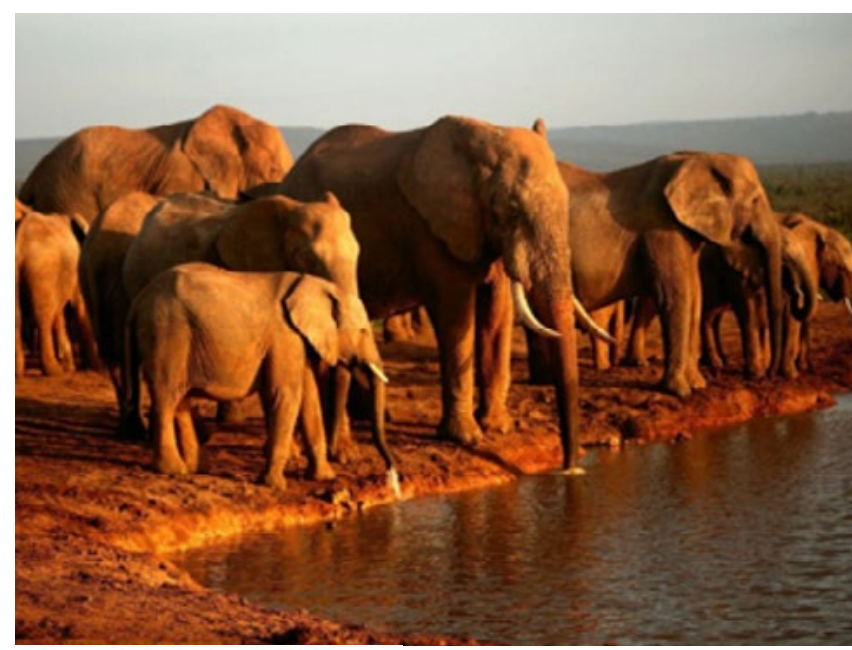

Figura 1: Manada de elefantes Fuente: (MININ, 2019)

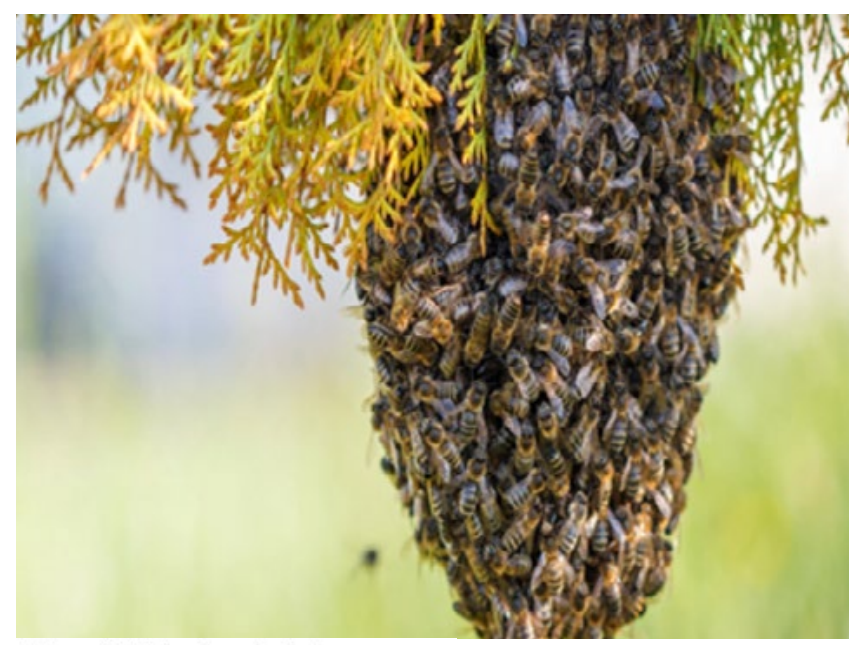

Figura 2: Enjambre de abejas

Fuente: (Comunidad de Madrid, 2018)

\section{Comportamiento del Centro Democrático: Enjambre de abejas}

Un enjambre no es más que un grupo de abejas que viven dentro de una colmena la cual cumple un rol muy importante para la supervivencia y obtención de nutrientes. Dentro de las colmenas se da una división natural de las tareas en donde cada abeja cumple una función importante con el objetivo de que el enjambre crezca y sea seguro principalmente para la reina. Al interior del enjambre se encuentran primero las abejas obreras las cuales son las encargadas de construir y expandir la colmena, segundo las abejas zánganas encargadas de fecundar a la reina y por úl- 
timo la reina, que cumple el papel de dirigir el rumbo del enjambre (Ordóñez, 2010).

Este tipo de comportamiento se puede relacionar con el partido Centro democrático debido a que se tiene las siguientes semejanzas: Primero se cuenta con una abeja reina que en este caso se puede asociar con el papel que tiene el expresidente Álvaro Uribe dentro del partido, es evidente que el exmandatario decide el rumbo del partido, que políticas apoyar y a que candidatos apoyar o no, en el contexto del 2014 a 2018 fue notable la oposición creada por el entonces senador Uribe y apoyada por el centro democrático al expresidente Santos, por ejemplo durante este periodo se llevó a cabo las negociaciones de paz liderado por el expresidente Santos: durante este proceso el exmandatario contó con casi un apoyo totalitario de los partidos, exceptuando el centro democrático, esta decisión se dio principalmente por la oposición visible y el desacuerdo del entonces senador Álvaro Uribe.

Segundo se puede resaltar que dentro del partido existe una estructura jerárquica, en donde no solo se tiene una abeja reina, sino que también cuenta con abejas zánganas este trabajo es realizado por la población que apoya el interés de este partido y los miembros del centro democrático. Por último, están las abejas obreras en este papel se puede asociar con el trabajo que realizan los senadores miembros del partido ya que ellos se encargan de proteger y apoyar los ideales del expresidente Álvaro Uribe.

\section{Comportamiento del Polo Democrático Al- ternativo: Osos polares}

Los osos polares son animales solitarios, solo se reúnen en momentos de apareamiento, debido a esto su comportamiento no es homogéneo y varía de un individuo al otro. No obstante existen conductas que comparten estos grandes mamíferos entre ellas el de no ser animales territoriales debido a que no se establecen en un solo espacio sino que prefieren estar en continuo movimiento el cual está determinado por el ambiente y su constante curiosidad de explorar nue- vos lugares; otra conducta es que a diferencia de otros animales su comportamiento no se basa principalmente en la búsqueda de alimento, por otra parte, son cautelosos en su entorno y no necesitan de la protección de otras especies (National Geographic España, 2020).

El Polo Democrático es un partido que comparte conductas con los osos polares, esto se evidenció durante el periodo 2014-2018; en el cual el partido mantuvo un comportamiento estable en sus acciones, sin importar quien estuviera en el poder es decir quién era el jefe de estado: por otra parte el Polo Democrático se ha caracterizado por mantenerse al margen construyendo una oposición de izquierda contra el gobierno, creando una imagen fuerte que le ha permitido sobrevivir en el sistema político sin necesidad de estar en una búsqueda constante de protección. Estas conductas se asocian con los osos polares que es fuerte, solitario, que sin importar los animales que lo rodean su comportamiento no se ve afectado y logran sobrevivir en ambientes difíciles sin necesidad de aliarse con otras especies.

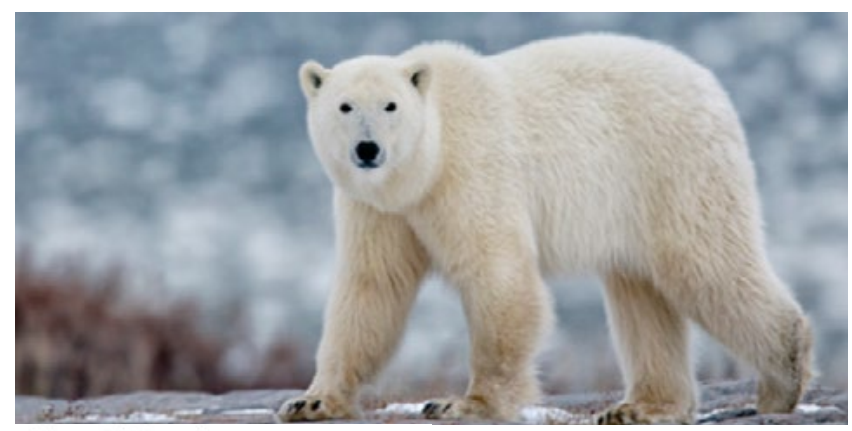

Figura 3: Oso polar

Fuente: (National Geographic, 2019)

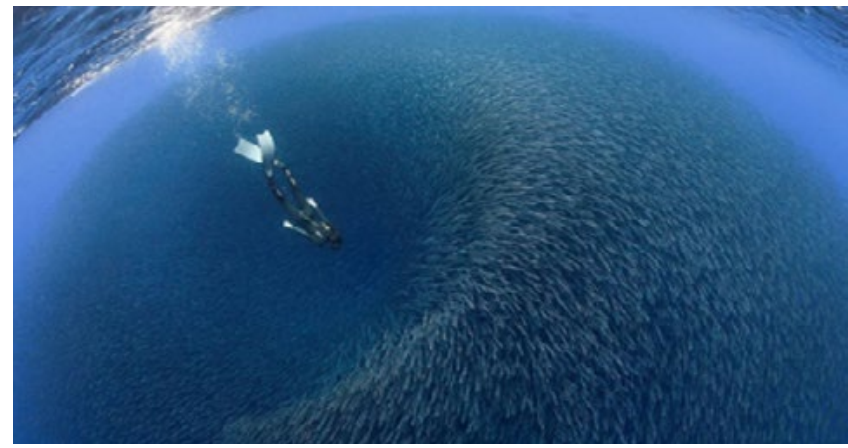

Figura 4: Cardumen de peces

Fuente: (National Geographic, 2015) 
4. Comportamiento del Movimiento Alternativo Indígena y Social: Cardumen de Peces

Los cardúmenes son un conjunto de peces de similar tamaño, no deben ser de su misma especie y que tengan la misma orientación es decir que se dirijan al mismo lugar. Generalmente la población de los cardúmenes aumenta a lo largo del trayecto debido a que diferentes peces que cumplan con las características anteriormente mencionadas se les unen: este tipo de conducta tiene como beneficio la protección y defensa contra los depredadores.

El comportamiento del partido Maís se asocia con la conducta de los cardúmenes debido a que tiene las siguientes características: primero la mayoría de los miembros del partido son de alguna comunidad indígena esto se puede asociar con agregación de peces con características similares; segundo entre los objetivos de este partido tiene el de defender los derechos de los indígenas y entre mayor sean la cantidad de miembro más es fácil les es conseguir este objetivo; además el de tener mayor peso político, esto se relaciona con los beneficios de protección de los cardúmenes.

\section{Simbiosis, mutualismo}

La simbiosis "es la relación habitual entre dos o más especies, que redunda en beneficio de todas ellas" (Hilje, 1984, p. 58), dentro de esta conducta hay dos categorías las cuales son: recíproca y antagónica, en este caso tomaremos la primera. La simbiosis recíproca se refiere a que no hay daño entre las especies, en esta categoría se encuentra el mutualismo que es "la relación entre individuos de dos o más especies, de la cual temporal o permanentemente todos obtienen beneficios indispensables para su existencia” (Hilje, 1984, p. 58). Un ejemplo de esta conducta es:

\subsection{Comportamiento del partido Cambio Radi- cal y el Liberal: El pez payaso y la anémona}

El pez payaso es inmune al veneno de las anémonas, a raíz de esto puede vivir en ellas y a su vez ocul- tarse de los peces grandes, además mantiene limpia la anémona eliminando parásitos y atrae alimento para esta. En este caso los partidos como el Liberal y Cambio Radical cumplen con este comportamiento el cual se da una relación de servicio-servicio dado que durante la presidencia de Juan Manuel Santos y los partidos políticos han mantenido un vínculo de ayuda mutua, es decir que los dos se benefician por favores que se ofrecen como por ejemplo, otorgando cargos en el gabinete e incluso la vicepresidencia como se dio con el Cambio Radical y estos por igual dan un servicio como sucedió con el apoyo al Proceso de Paz o en el mismo Senado.

\subsection{Comportamiento de la Alianza verde y Op- ción ciudadana: Pica buey y el búfalo del cabo}

El pica buey y el búfalo del cabo se benefician mutuamente debido a que el ave se alimenta de los parásitos como piojos o garrapatas que tiene el búfalo en su lomo y este a su vez protege al ave de posibles atacantes; la relación que tienen es de servicio-recurso. En este caso se relaciona con los partidos de la Alianza Verde y Opción Ciudadana en el que uno se beneficia de un recurso y ofrece un servicio, es decir que uno de estos obtiene ganancias (como por ejemplo el alimento como en el caso del pica buey) y solo dio algo para beneficiar al otro.

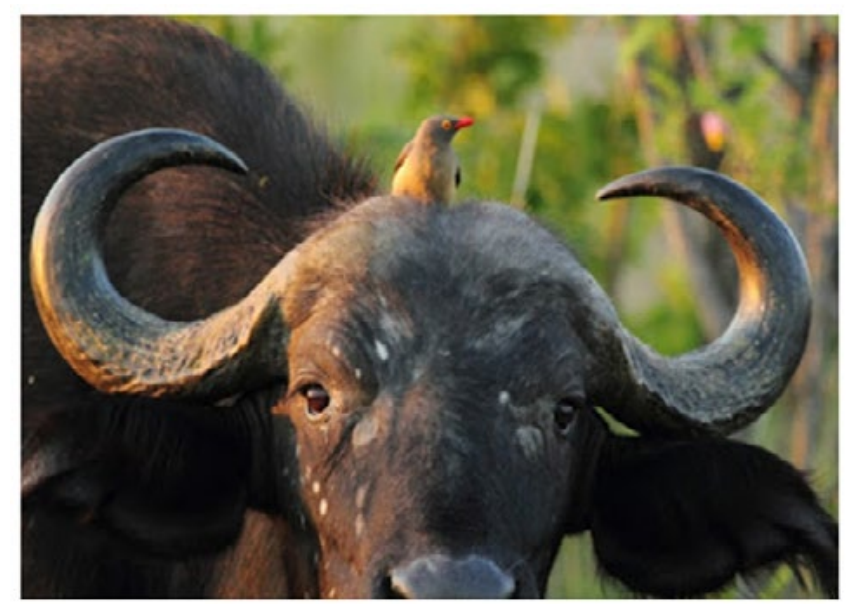

Figura 5: Relación simbiótica de pica bueyes y búfalo del cabo Fuente: (Manuel, Liso \& Fernández, 2017) 


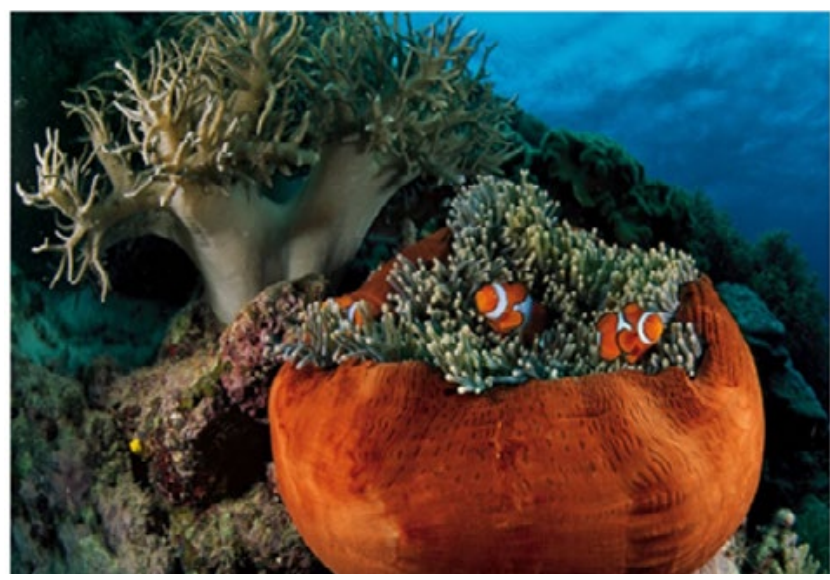

Figura 6: Pez payaso y la anémona Fuente: (National Geographic, 2010)

\section{Comportamiento del partido Conservador: Jauría de lobos}

Las jaurías de lobos en su posición de macho alfa y dominancia, según Mech (1999), los lobos en cautiverio suelen generar una jerarquía de dominancia en el que se necesita un alfa, en el que puede ser un "macho alfa" o una "hembra alfa", este rango solo lo tienen los animales reproductores; en cambio, los lobos en libertad tienen una tendencia de solo procrear una familia y algunas veces adoptar a otros miembros, pero estos no perduran por mucho tiempo.

Asimismo, según Candelas (2017) los "macho alfa" deben mantener su poderío no sólo por medio del uso de la fuerza, sino también lo hacen a través del populismo, es decir, que si sus subalternos consideran su comportamiento de manera tiránica o que no protege lajauría de la manera correcta estos pueden rebelarse y a su vez derrocar al "macho alfa"

Esta conducta de las jaurías se ve reflejado en el partido Conservador ya que el comportamiento de las manadas puede variar debido a dos factores importantes: primero, la necesidad de obtener o buscar los mayores recursos y segundo, garantizar su supervivencia. Con respecto a estos dos factores el partido Conservador ha garantizado su supervivencia bien sea por medio del clientelismo o cuando no se consigue sus objetivos va en busca de un nuevo líder al cual puede seguir para que le brinde seguridad; tal como se evidencio en las elecciones del 2014 donde presentaron su propio candidato a la presidencia y no apoyaron la reelección, pero a pesar de esto obtuvieron participación en el gabinete del expresidente Santos y apoyaron el Proceso de Paz, el cual fue crucial.

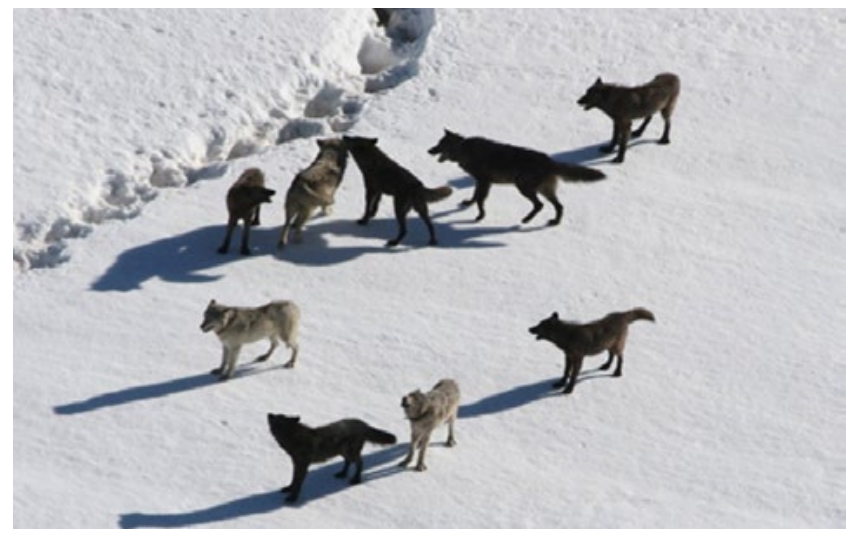

Figura 7: Jauría de lobos

Fuente: (National Park Service, 2020)

A partir del enfoque biopolítico se logra observar los distintos comportamientos de los animales y su ambiente, asimismo se puede explicar de mejor manera la conducta que tienen los partidos políticos en el Senado colombiano durante el periodo 2014-2018 y así determinar la verdadera razón por la que actúan estos mismos. Se debe agregar que el ambiente en el cual habitan las diferentes especies del reino animal son variadas, algunas veces estas mismas cambian como consecuencia a los cambios climáticos, las estaciones del año, la escasez de comida, la presencia de depredadores, entre otros. Esto sucede con el ambiente político del Senado que está influenciado por cambios en su entorno como escándalos de corrupción, temas mediáticos, la oposición u apoyo del Proceso de Paz, la unión de partidos políticos, el cambio de presidencia tanto del senado como del país, entre otros. "Cuando interesa que alguien nos ame, le presentamos lo mejor de nosotros, lo conquistamos". (Trujillo, 2018)

\section{Conclusión}

El enfoque biopolítico es poco implementado en 
la ciencia política visto que se ha generado gran confusión de ¿qqué es? y para qué sirve, pero a pesar de que a través de los años se ha generado definiciones contrapuestas de este término, la biopolítica científica es una herramienta que debería implementarse con mayor frecuencia en el estudio del comportamiento político. En este caso se analizó el comportamiento de los partidos políticos que hicieron parte del senado colombiano en el periodo 2014-2018, con diferentes conductas animales con el fin de poder determinar el porqué de su comportamiento.

Como resultado de la anterior investigación exploratoria se puede destacar: En primer lugar, los partidos políticos no basan sus acciones solamente en su ideología, sino que existen diferentes factores que influyen en su comportamiento como la búsqueda de recursos políticos, la búsqueda de protección, los beneficios económicos, aumentar su poder político e influencia: esto se asemeja al comportamiento de los animales los cuales determinan su comportamiento según sus necesidades. Segundo, existen similitudes en la organización de los diferentes grupos de animales con la estructura de los partidos políticos. Tercero, se puede destacar la importancia del ambiente en el comportamiento tanto de los animales como de los partidos políticos, a causa de que los distintos cambios o estímulos que reciben en su entorno puede cambiar las dinámicas y los comportamientos. En el caso del senado colombiano el ambiente político cambia según la persona que esté en la presidencia, el clima político, los diferentes conflictos que surjan o las soluciones que se busquen para los conflictos.

Es importante resaltar que el comportamiento de un partido puede variar conforme al ambiente político es decir, según su entorno los partidos políticos adaptan distintas conductas de animales por ejemplo, un partido que en un periodo asumió una actitud de manda de elefantes debido a que su ambiente le ofrecía todo lo que necesitaba y podía mantener una actitud despreocupada, al siguiente periodo este entorno cambia y se vuelve más hostil; el partido político puede aplicar una conducta de jauría de lobos en donde busca al líder más fuerte con el fin de conseguir protección y asegurar su supervivencia.

Finalmente destacar que en todo sistema lo más relevante es la supervivencia sobre las otras especies, en donde es importante tener inteligencia, fuerza adaptabilidad, versatilidad e implementar las mejores estrategias con el fin de poder alcanzar los objetivos propuestos y lograr los intereses de las distintas conductas adaptadas de acuerdo al ambiente en que se encuentre. 


\section{Referencias}

Borge, D. (2017). El omega de la aurora. España: megustaleer.

Candelas, M. (2017) Selva y poder: Lecciones de ciencia política en el reino animal. Asociación de Comunicación Política. Recuperado de: https://compolitica.com/selva-y-poder-lecciones-de-ciencia-politica-en-el-reino-animal/

Comunidad de Madrid. (14 de Julio de 2018). Los Bomberos de la Comunidad realizan este año más de 900 intervenciones por enjambres de abejas. Comunidad de Madrid. Obtenido de https://www.comunidad.madrid/noticias/2018/07/14/bomberos-comunidad-realizan-este-ano-900-intervenciones-enjambres-abejas

Congreso de Colombia 2014-2018. (2014). Revista Semana. Recuperado de: https://especiales.semana.com/especiales/congreso-colombia-2014-2018/

Congreso de la República. (17 de junio de 1992). Reglamento del Congreso; el Senado y la Cámara de Representantes. (Ley 5 de 1992). DO: 40.483

Congreso de la Republica. (24 de marzo de 1992). normas sobre las Comisiones del Congreso de Colombia y se dictan otras disposiciones. (Ley 3 de 1992). DO: 40.390

Constitución política de Colombia (1991). Bogotá, Colombia: Leyer.

Flohr, H. (1990). Review of Roger D. Masters' "'The Nature of Politics". Politics and the Life Sciences, 9(1), 176-178. http://www. jstor.org/stable/4235747

Gunneflo, M. (2015). Rudolf Kjellén: la biopolítica nórdica ante el estado del bienestar. Retfærd: Nordisk juridisk tidsskrift, 35 (3). http://retfaerd.org/retfaerd-nr-150-20153/rudolf-kjellen-nordic-biopolitics-before-the-welfare-state/

Herrera Burton, D. (2018). Participación política: entre normatividad (Habermas y Rawls) y biopolítica (Hardt y Negri). Disponible en http://repositorio.uchile.cl/handle/2250/170138

Hilje, L. (1984). Simbiosis: Consideraciones terminológicas y evolutivas. Uniciencia, 1(1), 57-60. Recuperado de: https://dialnet. unirioja.es/servlet/articulo?codigo $=5381266$

Manuel, M., Liso, A., \& Fernández, S. (21 de marzo de 2018). Pajareando por Huesca. Obtenido de http://pajareandoporhuesca. blogspot.com/2018/03/sudafrica-17-de-noviembre-al-6-de. html

Marino, Y.(1996). Comportamiento animal y conducta humana. Psicothema 8(Sup) 149-163.

Mech, D. (1999). Alpha status, dominance, and division of labor in wolf packs. Canadian Journal of Zoology 77.1196-1203.

MININ, E. D. (8 de abril de 2019). Un elefante mata a un cazador furtivo y su cuerpo es devorado por los leones. Obtenido de: https://cadenaser.com/ser/2019/04/08/internacional/1554701417_531724.html

Misión de Observación Electoral (MOE) (2014) Resultados Electorales Congreso 2014. Recuperado de: https://moe.org.co/ wp-content/uploads/2017/05/Libro-Resultados-Electorales-Congreso-2014-2.pdf

National Geographic España. (2020). National Geographic España. Obtenido de National Geographic España: https://www.nationalgeographic.com.es/animales/osos-polares

National Geographic. (26 de febrero de 2010). National Geographic. Obtenido de https:/www.nationalgeographic.es/animales/ pez-payaso-y-anemona

National Geographic. (4 de diciembre de 2015). Obtenido de https://www.nationalgeographic.com.es/mundo-ng/francis-perez-gana-el-prestigioso-por-el-planeta-en-la-categoria-subacuatica-2_9953

National Geographic. (9 de diciembre de 2019). Obtenido de https:// www.ngenespanol.com/el-mundo/56-osos-polares-avistaron-aldea-rusa-ryrkaypiy/

National Park Service. (2020). National Geographic. Obtenido de https://www.nationalgeographic.com.es/animales/lobos/fotos/1/9

Neira, H. (2014). Política y vida animal: la analogía del buen gobier- 
no. Scientiae Studia, 12(2), 261-284. https://doi.org/10.1590/ S1678-31662014000200003

Ordóñez, E. Z. (2010). Bases biológicas del comportamiento animaly humano. Bogotá, Colombia: Universidad Nacional de Colombia.

Rabinow, P. \& Rose, N. (2006). Biopower Today. Biosocieties, 1(2), 195-217.

Real Academia Española (2019). Diccionario de la lengua española (23. ${ }^{\text {a }}$ edición). Recuperado de: https://dle.rae.es/ambiente

Rodríguez Pico, C. R. (2016). Los partidos políticos colombianos ante los acuerdos de paz de La Habana. Universidad Nacional de Colombia, Facultad de Ciencias Económicas. Recuperado de: https://repositorio.unal.edu.co/bitstream/handle/ unal/58265/ClaraRoc\%c3\%adoRodr\%c3\%adguezP.2016.pdf?sequence $=1 \&$ isAllowed $=y$

Santos salió en defensa de la llamada 'mermelada. (21 de 3 de 2014). El espectador. Recuperado de: https://www.elespectador. com/noticias/politica/santos-salio-en-defensa-de-la-llamada-mermelada/

Semana (2014), Congreso de Colombia 2014-2018. Recuperado de https://especiales.semana.com/especiales/congreso-colombia-2014-2018/

Senado de la República de Colombia (2019). ¿Qué es y cómo está conformado el Congreso de la República? Recuperado de: http://www.senado.gov.co/index.php/component/content/ article/2-uncategorised/110-preguntas-frecuentes?Itemid $=101$

Somit, A. \& Peterson, S. (1998). Biopolitics after Three Decades - A Balance Sheet. British Journal of Political Science, 28(3), 55957l. Retrieved:http://www.jstor.org/stable/194269

Trujillo Culebro, F. (2018). La didáctica de la literatura en secundaria. Amauta, 16(32), 49-68. https://doi.org/10.15648/ am.32.2018.4.

2020, Vol. 13(26) 63-75 CThe Author(s) 2020 Reprints and permission: www.americana.edu.co http://publicaciones.americana.edu.co/index.php/pensamientoamericano/index 
